\title{
Architectural tunability of mechanical metamaterials in the nanometer range
}

\author{
Chantal Miriam Kurpiers ${ }^{1,2} \cdot$ Stefan Hengsbach ${ }^{3} \cdot$ Ruth Schwaiger ${ }^{4,5}$
}

Received: 5 May 2021 / Accepted: 20 June 2021 / Published online: 1 July 2021

(c) The Author(s) 2021

\begin{abstract}
Mechanical metamaterials can exhibit extraordinary mechanical properties due to a specific architecture rather than the base material. When the structural dimensions reach the sub-micrometer range, such micro- and nanolattices may also benefit from size-affected mechanical properties. However, well-defined geometric adjustments on this length scale are limited by the resolution limits of the underlying manufacturing technology. Here, we used a 3D direct laser writing (3D-DLW) process with integrated laser power variation to fabricate polymeric microlattices, which were then pyrolized to obtain glassy carbon structures. The laser power was varied by a quadratic function along the beams from one node to another over the length of a unit cell, thus enabling geometric adjustments in the range of a few nanometers. Rounded and notch-like joints were realized by increased and reduced laser power at the nodes, respectively. Furthermore, the beam cross section was varied along the beam length, thereby creating convex or concave beam shapes. A laser power variation opens up new design possibilities for micro- and nanolattices in the sub-micrometer range by overcoming process related limitations.
\end{abstract}

\section{Introduction}

Micro- and nanoarchitected materials, such as beam-, shell-, or plate-based lattices, have attracted increasing attention due to their often extraordinary mechanical properties, exceeding those of most known bulk materials [1,2]. The

Ruth Schwaiger was an editor of this journal during the review and decision stage. For the MRS Advances policy on review and publication of manuscripts authored by editors, please refer to mrs. org/editor-manuscripts.

Chantal Miriam Kurpiers

chantal.kurpiers@kit.edu

1 Institute for Applied Materials (IAM), Karlsruhe Institute of Technology (KIT), 76344 Eggenstein-Leopoldshafen, Germany

2 3DMM2O - Cluster of Excellence (EXC-2082/1 390761711), Karlsruhe Institute of Technology (KIT), 76131 Karlsruhe, Germany

3 Institute of Microstructure Technology (IMT), Karlsruhe Institute of Technology (KIT), 76344 Eggenstein-Leopoldshafen, Germany

4 Institute of Energy and Climate Research (IEK), Forschungszentrum Jülich, 52425 Juelich, Germany

5 Chair of Energy Engineering Materials, RWTH Aachen University, 52056 Aachen, Germany finest feature resolution can be achieved by 3D printing, while the printing techniques are mostly restricted to polymer-based systems [1]. Various coating deposition techniques can be used to add additional functionalities [3] through deposition of thin metallic and ceramic coatings with thickness in the (sub)nanometer range on nano- and microlattices. Recent work has emphasized the importance of architecture, for example for strength and stiffness as well as damping behaviors and recoverability [4-9]. However, adjustments of the geometries in the nanometer range are challenging, due to resolution limits of the respective 3D manufacturing technologies [10,11].

In this paper, we demonstrate a single step 3D direct laser writing (3D-DLW) method to vary structural elements of microlattices in the sub-micrometer range. Beam-based tetrahedral structures were investigated because their high specific strength has already been demonstrated [9]. By varying the laser power within structural elements, we are able to change beam and node shapes and manufacture variations of a structure but on the base of the same initial model. Subsequent pyrolysis converting and shrinking the polymeric microlattices to glassy carbon nanolattices enables further design freedom of nanometer sized structural elements. 


\section{Experimental}

Tetrahedral microlattices (Fig. 1) with different sizes were fabricated on silicon substrates using 3D-DLW (Photonic Professional GT2, Nanoscribe GmbH, Eggenstein-Leopoldshafen, Germany) and the proprietary photoresist IP-Dip (Nanoscribe $\mathrm{GmbH}$ ). After printing and development, the fine structures were dried in a critical point dryer (EM CPD300, Leica Microsystems GmbH, Wetzlar, Germany) to avoid distortion or collapse due to surface tension. Pyrolysis of the polymeric structures was performed in a vacuum tube furnace at a pressure of $10^{-6}$ to $10^{-5} \mathrm{mbar}$ for one hour at a maximum pyrolysis temperature of $900{ }^{\circ} \mathrm{C}$. During the process the polymeric microlattices underwent a shrinkage of around $80 \%$ in size and were converted to glassy carbon (GC). The sample dimensions before and after pyrolysis were measured using a helium ion microscope (HIM, ORION NanoFab, Zeiss, Oberkochen, Germany) and a scanning electron microscope (SEM, SUPRA V-60, Zeiss, Oberkochen, Germany).

The software MATLAB (Version R2019b, The MathWorks, Inc., Natick, USA) was used to define the printing parameters and printing sequence as well as the dimensions. The structures were printed layer-by-layer with a constant distance between two adjacent voxel lines in the $\mathrm{x}$ - and $\mathrm{y}$-directions (hatching distance) and $\mathrm{z}$-direction (slicing distance) of $200 \mathrm{~nm}$ using the galvo scanning mode. The inclined and horizontal beams show a circular cross section. The tetrahedral structures were printed with a laser power (LP) ranging between $10 \%(2.65 \mathrm{~mW})$ and $50 \%(12.9 \mathrm{~mW})$ of the available LP of the Nanoscribe system, and a constant printing speed of $3000 \mu \mathrm{m} \mathrm{s}^{-1}$. LP variations within the structural elements, as shown schematically in Fig. 1a, b, were applied using the naming scheme LP $a-b$. Here, $a$ corresponds to the LP in the nodes and $b$ to the LP in the middle of the beams. This change in LP from one node to another over a beam was applied following a quadratic function and was implemented into the
MATLAB code by allocating a LP value to every point of the geometry [12].

The tetrahedral structure size was varied by different sizes of the edge length $T$ of a unit cell (Fig. 1c), which is equivalent to the beam length. Sizes of $7.5 \mu \mathrm{m}$ and $10 \mu \mathrm{m}$ were used and henceforth labelled as T7.5 and T10. To measure the lattice dimensions and relative densities of the architectures, successive cross sections with a step size of $50 \mathrm{~nm}$ were performed with a dual beam focused ion beam microscope (FIB, Helios NanoLab DualBeam 650, ThermoFisher Scientific, Hillsboro, USA).

\section{Results}

To realize a nanoscale modification of geometry in glassy carbon nanolattices, a 3D-DLW process with a variable LP was designed (Fig. 1a). Without a modification of the geometric model, by changing the LP during writing, different shapes of nodes and beams were produced. The overall lattice dimensions of both, the polymeric and glassy carbon structures depend on the absolute value of the LP and on the LP difference from node to middle of a beam. Figure 2 shows T10 tetrahedral structures with constant writing parameters, labelled as LP40-40 (Fig. 2a, b) and with LP variation, i.e. LP10-40 (Fig. 2c, d). The polymeric structure LP40-40 (Fig. 2a) exhibited no measurable size reduction in the diameter $D$ compared to the input dimensions, but a $2 \%$ reduction in the height $H$ regarding the input dimensions. By contrast, the LP10-40 structure (Fig. 2c) exhibited a reduction of $6 \%$ in diameter and height. Regarding pyrolysis induced shrinkage, the LP10-40 structure exhibited a slightly higher shrinkage of one percentage point compared to LP40-40. Similar trends were observed for the T7.5 tetrahedral structures. The polymeric structure LP10-40 showed up to 4 percentage points higher shrinkage values of the structure dimensions compared to LP40-40. Similarly, due to pyrolysis, an increased shrinkage of LP10-40 of up to 3 percentage points was observed compared to LP40-40.
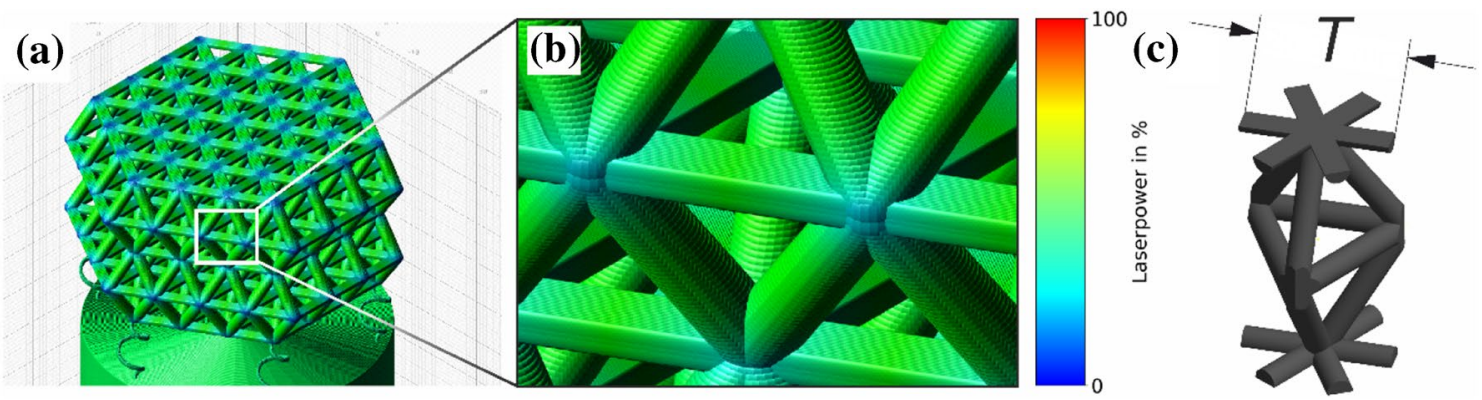

Fig. 1 a Model of tetrahedral structure with variable LP in the nodes and in the middle of the beams and $\mathbf{b}$ detail of the structure with corresponding color coding of the LP of the Nanoscribe system in percent. $\mathbf{c}$ Tetrahedral unit cell with unit cell edge length $\mathrm{T}$ 

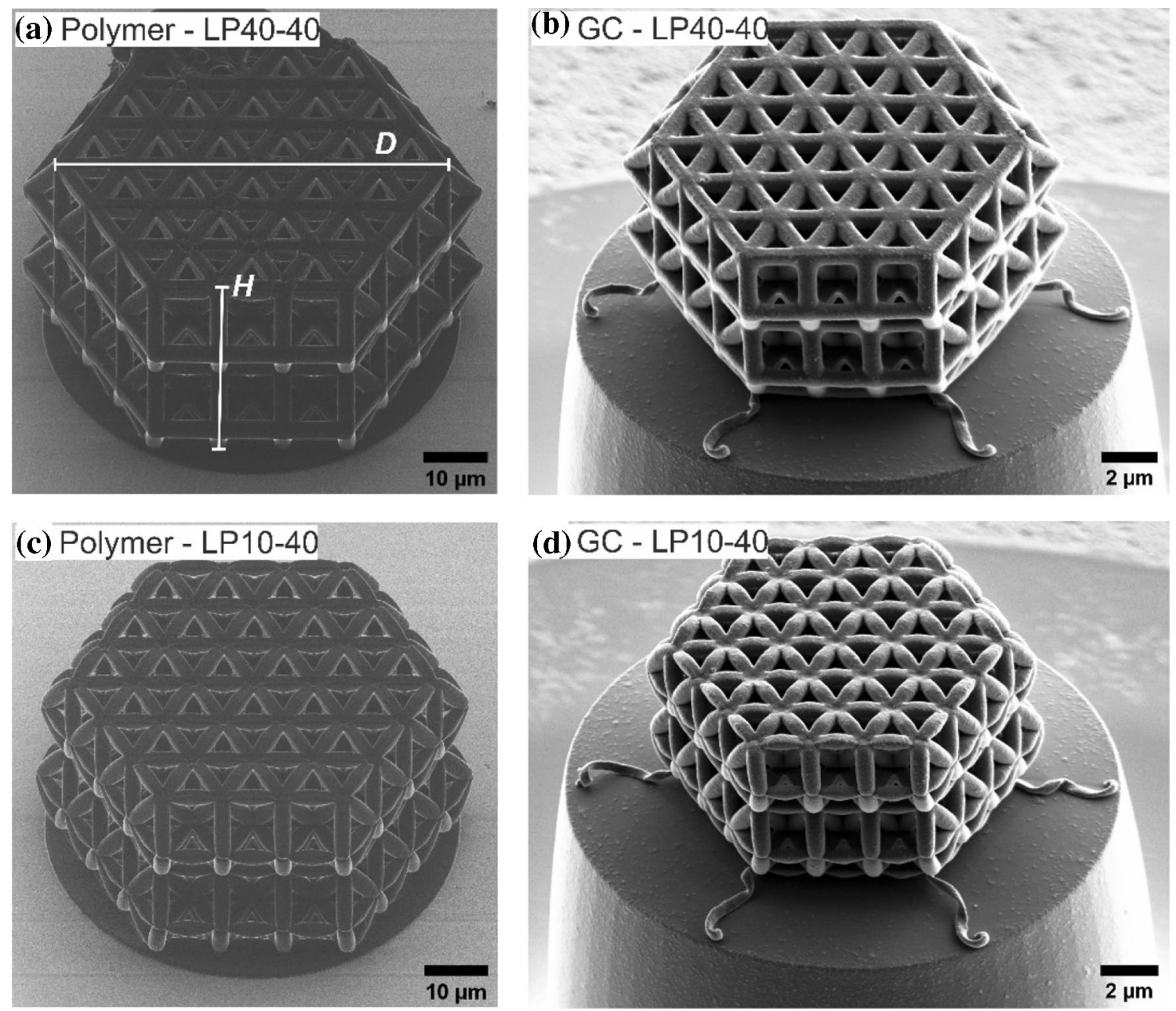

Fig. 2 Micrographs of typical tetrahedral structures T10. a constant LP as-written, polymeric structure and $\mathbf{b}$ the corresponding pyrolized GC structure. $\mathbf{c}$ variable LP as-written, polymeric structure and $\mathbf{d}$ the

In addition to the differences in the overall shrinkage, a modification of the joint shape was observed when the LP was varied. As can be seen in Fig. 3 for LP40-40 (Fig. 3a, b), a constant LP resulted in rounded joints at the nodes, which is also apparent for the LP40-30 structure (Fig. 3g, h) with a higher LP in the nodes than in the beams. By contrast, the LP variations in Fig. 3c-f, with reduced laser power at the nodes, produced rather sharp notches at the joints. Furthermore, the shape of the beams was affected by the LP variation. While the constant LP resulted in beams with a constant cross section (LP40-40 in Fig. 3a, b), increased LP towards the middle of the beam length created convex beams (LP10-40 and LP20-50 in Fig. 3c-f). Using the T7.5 LP10-40 structure (Fig. 3d) as an example, a difference of less than $30 \mathrm{~nm}$ between beam diameter in the middle of the beams and beam diameter at the joints was realized. Conversely, when the LP in the beams was lower than in the nodes, the beams were more slender towards the middle corresponding pyrolized GC structure. Polymeric structures (a) and (c) were imaged using the helium ion microscope and GC structures (b) and (d) using the scanning electron microscope

(LP40-30 in Fig. $3 \mathrm{~g}, \mathrm{~h}$ ). For the $10 \mu \mathrm{m}$ unit cell, a LP of $40 \%$ in the middle of the beams resulted in a beam diameter of $596 \pm 9 \mathrm{~nm}$ for LP40-40 and $579 \pm 5 \mathrm{~nm}$ for LP10-40. For higher and lower LP, i.e. 50\% and 30\%, the diameters were $627 \pm 7 \mathrm{~nm}$ and $541 \pm 10 \mathrm{~nm}$ for LP20-50 and LP40-30, respectively. Similarly, for the smaller structures with $7.5 \mu \mathrm{m}$ unit cell, the beam diameters were affected by the LP. A LP of $40 \%$ resulted in a diameter of $477 \pm 7 \mathrm{~nm}$ for LP40-40 and $456 \pm 4 \mathrm{~nm}$ for LP10-40, a LP of $50 \%$ in a diameter of $501 \pm 10 \mathrm{~nm}$ (LP20-50), and a LP of $30 \%$ in a diameter of $445 \pm 11 \mathrm{~nm}$ (LP40-30).

To study the interior structure of the nanolattices, we prepared FIB cross sections of the GC structures. In Fig. 4, LP40-40 and LP10-40 of the structures T10 are compared. The detail images of cross sections through the nodes clearly show the differences of the beam and node geometries for the different writing strategies, i.e. constant cross sections of the vertical beams (Fig. 4a) and a cross section variation 

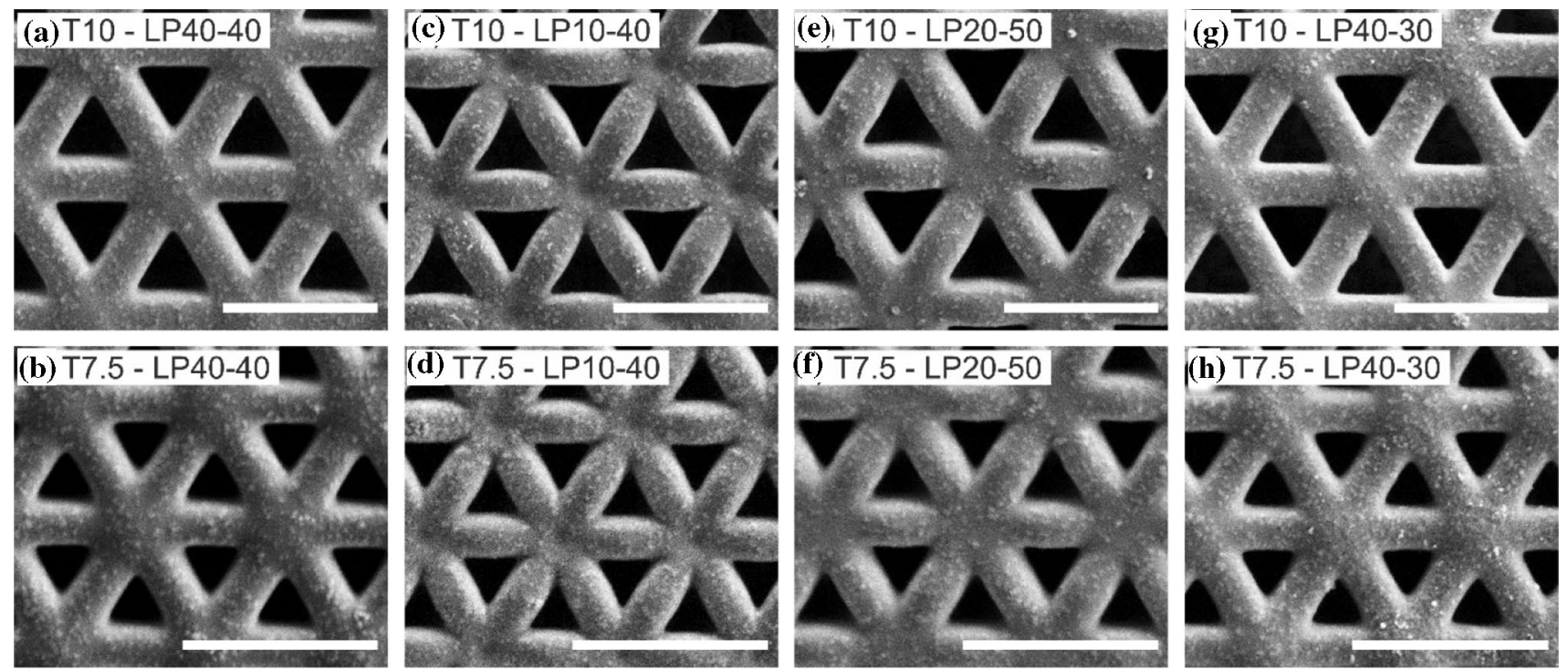

Fig. 3 Top view (SEM) of T10 and T7.5 GC microlattices produced from polymeric structures written with (a) and (b) constant LP40-40, (c) and (d) LP variation LP10-40, (e) and (f) LP variation LP20-50 and (g) and (h) LP variation LP40-30. All scale bars are $2 \mu \mathrm{m}$
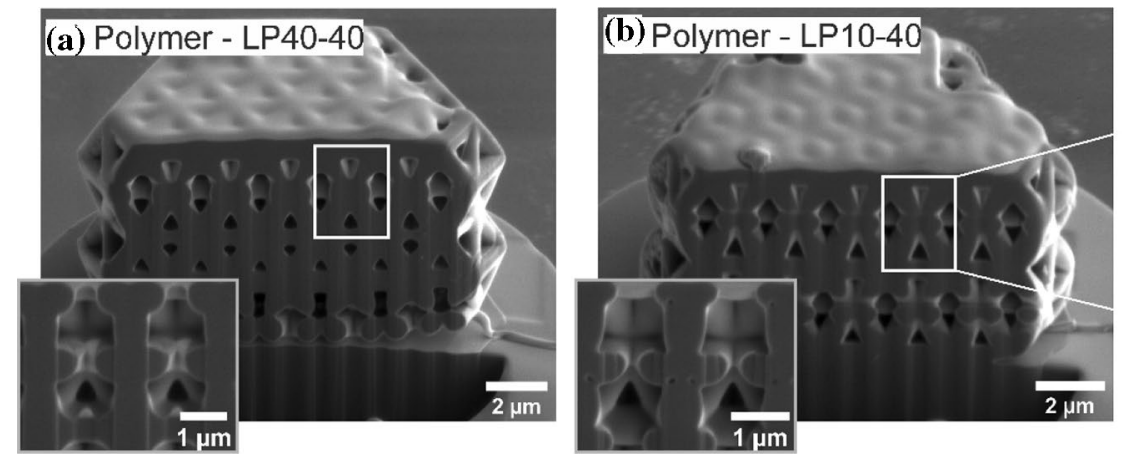

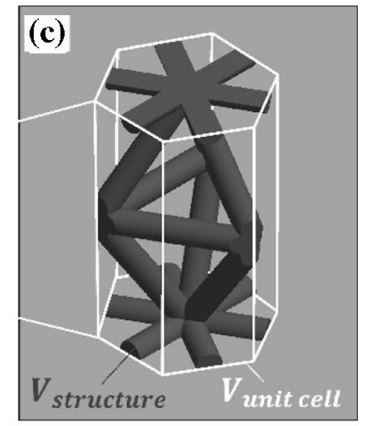

gles mark the unit cells. c Unit cell of the tetrahedral structure (indicated by the white rectangles in $\mathbf{a}$ and $\mathbf{b})$. The volume of the structure $V_{\text {structure }}$ is shown in gray and the volume of the unit cell $V_{\text {unit cell }}$ corresponds to the white geometry

density was estimated according to Eq. 1 . We observed a denser architecture for LP40-40 (Fig. 4a) of 2 percentage points compared to LP10-40 with reduced LP at the nodes (Fig. 4b).

\section{Discussion}

Various approaches have been described in order to tailor the properties of mechanical metamaterials by changing the architectural design [13-16]. However, solutions for locally tailored dimensions at sub-micrometer length scales have not yet been studied. By varying the laser power within the structural elements of micro- and nanolattices, the dimensions and the shapes of, e.g., the beams and nodes can be 
defined without additional process steps or the creation of new architectural models. In this study, within the given laser power range, the beams and nodes of polymeric microlattices and GC nanolattices were varied by several hundreds and several tens of nanometers, respectively. This facilitates additional design freedom for mechanical metamaterials.

Polymeric lattices were varied in size as well as beam and node shape by introducing a variable LP in the structural elements. These differences can be attributed to different degrees of conversion during the 3D-DLW process caused by the different energy input $[11,17]$. Since the hatching and slicing distances were kept constant at $200 \mathrm{~nm}$, greater overlap of successive voxel lines may occur for higher LP. In addition, due to the intersection of inclined and horizontal beams, the degree of polymerization at nodal points is different, which affects the resulting volume [17]. As shown for the T7.5 LP10-40 structure, a variable cross section along the beams was observed by introducing a LP variation with a reduced LP at the nodes (Fig. 4b). Small adjustments, such as a difference between beam diameter in the middle and beam diameter at the joints of less than $30 \mathrm{~nm}$ were realized, resulting in convex-shaped beams. Additionally, sharp notches at the joints are apparent for structures with a LP variation of LP10-40 (Fig. 3c, d). The pyrolysis appears to further reduce the node volume by size-dependent shrinkage. Here, an enhanced shrinkage of smaller structures or features is related to an enhanced surface-to-volume ratio and therefore to easier degassing during pyrolysis $[18,19]$. Applied to our structures, we suggest on the one hand a reduced size of polymeric LP-varied structures by a lower polymeric volume due to a reduced degree of conversion. On the other hand, for the GC structures we assume a higher shrinkage during pyrolysis related to an increased surface area, especially at the nodes with sharp notches having smaller volume. This can also explain the difference in the relative densities of the LP-varied structure LP10-40 (Fig. 4b) and the constant LP structure LP40-40 (Fig. 4a).

Furthermore, investigations of the mechanical performance of LP-varied structures are needed in order to fully understand the adjustability of mechanical properties by geometric changes. A number of multistep processing approaches for architectural adjustments of mechanical metamaterials have been reported recently $[7,16]$, either by creating new input models or by an additional process step after printing of the structures. Portela et al. [16] showed 3D-printed lattices with a unit cell length of $60 \mu \mathrm{m}$ and changed the node geometry by different input models to vary the mechanical properties. Due to process related size limitations $[10,11]$, reported design changes via 3D-DLW are in the micrometer range. Furthermore, systematic introduction of imperfections can cause targeted mechanical behaviour $[13,20]$, e.g., by missing beams. Integrating the presented LP variation in single unit cells or even single beams or nodes can generate a tailored deformation or failure behavior, which can be used to study the influence of imperfections on the mechanical performance.

With our approach of laser power variation in the 3D-DLW process, a design possibility for microlattices in the sub-micrometer range is accessible. Thus, design limitations regarding nanometer-scale variations of the geometry that are related to the printing technology used $[10,21]$ may be overcome. Additionally, we combine the LP variation with pyrolysis to achieve control over feature sizes of GC nanolattices in the nanometer range.

\section{Conclusion}

In this study, we introduce a method that facilitates the variation of beam and node shapes and dimensions in microlattices produced by 3D-DLW. Without the need for additional processing steps or different input models, the variation of laser power within structural elements facilitates specific joint shapes at the nanoscale as well as adjustments of the beam geometries. Using the example of a tetrahedral structure, polymeric microlattices were fabricated with the method of laser power variation, followed by a pyrolysis to obtain glassy carbon nanolattices. Depending on the applied laser power, joints were changed from rounded shape to sharp notches and beams with convex and concave shapes were realized. Especially at the nodes, a reduced laser power resulted in smaller dimensions of polymeric and glassy carbon tetrahedral structures. This might be related to a reduced polymerized volume during printing (3D-DLW) and a sizedependent shrinkage during pyrolysis.

Acknowledgments We would like to thank Christian Haug (Institute for Applied Materials (IAM-CMS), Karlsruhe Institute of Technology (KIT)) for support with the Auto Slice and View procedure at the FIB and the Karlsruhe Nano Micro Facility (KNMF, http://www.knmf.kit. edu) of the Karlsruhe Institute of Technology (KIT) for access to the 3D-DLW (2019-023-027489) and the helium ion microscope (2019023-027490). This project was financially supported by Germany's Excellence Strategy via the Excellence Cluster "3D Matter Made to Order" (EXC-2082/1-390761711).

Funding Open Access funding enabled and organized by Projekt DEAL.

Data availability The datasets generated during and/or analyzed during the current study are available from the corresponding author on reasonable request.

\section{Declarations}

Conflict of interest The authors declare no conflict of interest.

Open Access This article is licensed under a Creative Commons Attribution 4.0 International License, which permits use, sharing, 
adaptation, distribution and reproduction in any medium or format, as long as you give appropriate credit to the original author(s) and the source, provide a link to the Creative Commons licence, and indicate if changes were made. The images or other third party material in this article are included in the article's Creative Commons licence, unless indicated otherwise in a credit line to the material. If material is not included in the article's Creative Commons licence and your intended use is not permitted by statutory regulation or exceeds the permitted use, you will need to obtain permission directly from the copyright holder. To view a copy of this licence, visit http://creativecommons. org/licenses/by/4.0/.

\section{References}

1. J. Bauer, L.R. Meza, T.A. Schaedler, R. Schwaiger, X. Zheng, L. Valdevit, Nanolattices: an emerging class of mechanical metamaterials. Adv. Mater. (2017). https://doi.org/10.1002/adma.20170 1850

2. R. Schwaiger, L.R. Meza, X. Li, The extreme mechanics of micro- and nanoarchitected materials. MRS Bull. 44(10), 758-765 (2019). https://doi.org/10.1557/mrs.2019.230

3. A.R. Garcia-Taormina, A. Alwen, R. Schwaiger, A.M. Hodge, A review of coated nano- and micro-lattice materials. J. Mater. Res. 44, 782 (2021). https://doi.org/10.1557/s43578-021-00178-6

4. J. Bauer, C. Crook, A. Guell Izard, Z.C. Eckel, N. Ruvalcaba, T.A. Schaedler, L. Valdevit, Additive manufacturing of ductile, ultrastrong polymer-derived nanoceramics. Matter 1(6), 1547-1556 (2019). https://doi.org/10.1016/j.matt.2019.09.009

5. J. Rys, S. Steenhusen, C. Schumacher, C. Cronauer, C. Daraio, Locally addressable material properties in 3D micro-architectures. Extreme Mech. Lett. 28, 31-36 (2019). https://doi.org/10.1016/j. eml.2019.02.001

6. C. Crook, J. Bauer, A.G. Izard, C.S. de Oliveira, J.M. de Souza E Silva, J.B. Berger, L. Valdevit, Plate-nanolattices at the theoretical limit of stiffness and strength. Nat. Commun. 11(1), 1579 (2020). https://doi.org/10.1038/s41467-020-15434-2

7. Z. Vangelatos, L. Wang, C.P. Grigoropoulos, Laser pyrolysis for controlled morphing and chemical modification on 3D microlattices. J. Micromech. Microeng. 30(5), 55008 (2020). https://doi. org/10.1088/1361-6439/ab7c7e

8. E.D. Lemma, F. Rizzi, T. Dattoma, B. Spagnolo, L. Sileo, A. Qualtiei, M. de Vittorio, F. Pisanello, Mechanical properties tunability of three-dimensional polymeric structures in two-photon lithography Nanotechnol. IEEE Trans. (2016) https://doi.org/10. 1109/TNANO.2016.2625820

9. A. Schroer, J.M. Wheeler, R. Schwaiger, Deformation behavior and energy absorption capability of polymer and ceramic-polymer composite microlattices under cyclic loading. J. Mater. Res. 33(3), 274-289 (2018). https://doi.org/10.1557/jmr.2017.485

10. J. Bauer, A. Schroer, R. Schwaiger, O. Kraft, Approaching theoretical strength in glassy carbon nanolattices Mater. Nat. (2016). https://doi.org/10.1038/NMAT4561

11. J. Fischer, M. Wegener, Three-dimensional direct laser writing inspired by stimulated-emission-depletion microscopy [Invited]. Opt. Mater. Express 1(4), 614 (2011). https://doi.org/10.1364/ OME.1.000614

12. Nanoscribe GmbH, Photonic Professional (GT) User Manual.

13. Z. Vangelatos, K. Komvopoulos, C.P. Grigoropoulos, Vacancies for controlling the behavior of microstructured three-dimensional mechanical metamaterials. Math. Mech. Solids 24(2), 511-524 (2019). https://doi.org/10.1177/1081286518810739

14. M.S. Saleh, C. Hu, J. Brenneman, A.M. Al Mutairi, R. Panat, 3D printed three-dimensional metallic microlattices with controlled and tunable mechanical properties. Addit. Manuf. (2021). https:// doi.org/10.1016/j.addma.2021.101856

15. L.R.M. Meza, G.P. Phlipot, C.M. Portela, A. Maggi, L.C. Montemayor, A. Comella, D.M. Kochmann, J.R. Greer, Reexamining the mechanical property space of three-dimensional lattice architectures. Acta Mater. 140, 424-432 (2017). https://doi.org/ 10.1016/j.actamat.2017.08.052

16. C.M. Portela, J.R. Greer, D.M. Kochmann, Impact of node geometry on the effective stiffness of non-slender three-dimensional truss lattice architectures. Extreme Mech. Lett. 22(6058), 138-148 (2018). https://doi.org/10.1016/j.eml.2018.06.004

17. J. Bauer, A.G. Izard, Y. Zhang, T. Baldacchini, L. Valdevit, Programmable mechanical properties of two-photon polymerized materials: from nanowires to bulk. Adv. Mater. Technol. 4(9), 1900146 (2019). https://doi.org/10.1002/admt.201900146

18. B. Cardenas-Benitez, C. Eschenbaum, D. Mager, J.G. Korvink, M.J. Madou, U. Lemmer, I. De Leon, S.O. Martinez-Chapa, Pyrolysis-induced shrinking of three-dimensional structures fabricated by two-photon polymerization: experiment and theoretical model. Microsyst. Nanoeng. 5, 38 (2019). https://doi.org/10.1038/ s41378-019-0079-9

19. A. Albiez, R. Schwaiger, Size effect on the strength and deformation behavior of glassy carbon nanopillars. MRS Adv. 4(2), 133-138 (2019). https://doi.org/10.1557/adv.2018.648

20. T. Frenzel, C. Findeisen, M. Kadic, P. Gumbsch, M. Wegener, Tailored buckling microlattices as reusable light-weight shock absorbers". Adv. Mater. (Deerfield Beach, Fla) 28(28), 5865-5870 (2016). https://doi.org/10.1002/adma.201600610

21. J. Bauer, A. Schroer, R. Schwaiger, O. Kraft, The impact of size and loading direction on the strength of architected lattice materials. Adv. Eng. Mater. 18(9), 1537-1543 (2016). https://doi.org/10. 1002/adem.201600235 